\title{
Five Korean Cases of Respiratory Tract Infection by Filamentous Basidiomycetes
}

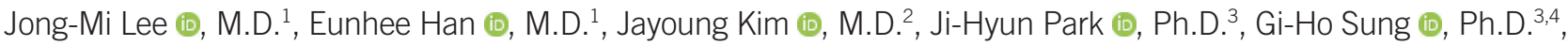 \\ Jong Hee Shin $\mathbb{1}$, M.D. ${ }^{5}$, and Yeon-Joon Park 무, M.D. ${ }^{1}$ \\ ${ }^{1}$ Department of Laboratory Medicine, College of Medicine, Seoul St. Mary's Hospital, Catholic University of Korea, Seoul, Korea; ${ }^{2}$ Department of Laboratory \\ Medicine and 'Institute for Healthcare and Life Sciences, International St. Mary's Hospital, College of Medicine, Catholic Kwandong University, Incheon, \\ Korea; ${ }^{4}$ Department of Microbiology, College of Medicine, Catholic Kwandong University, Gangneung, Korea; ${ }^{5}$ Department of Laboratory Medicine, Chonnam \\ National University School of Medicine, Gwangju, Korea
}

\section{Dear Editor,}

Invasive molds are the main cause of fungal diseases in immunocompromised patients; these diseases are diagnosed according to the guidelines of the European Organization for Research and Treatment of Cancer/Mycosis Study Group [1]. The mold species specified in these criteria are not clearly defined, but they usually include species with known pathogenic potential, such as Aspergillus, Fusarium, Mucorales, and Scedosporium spp. However, nonsporulating molds, especially basidiomycetes (BM), have been reported as emerging pathogens responsible for allergic and invasive diseases, most frequently involving the lungs [2]. As filamentous BM are white nonsporulating molds in culture, conventional identification is problematic. However, with recent advances in sequencing technologies, they have been reported as emerging pathogens [3]. Of the 218 global cases of human pathogenic BM, Schizophyllum commune is the most common (52\%), followed by Coprinopsis cinerea (5.9\%), Emmia lacerata (5\%), and a few cases of Irpex lacteus [2].

Although BM are increasingly identified in clinical specimens, little is known about their clinical significance, and Korean cases are rarely reported. We describe five Korean cases of respiratory infections caused by BM among patients admitted to the Seoul St. Mary's Hospital, Seoul, Korea, between June 2016 and July
2017. They were three cases of respiratory tract infection caused by $C$. cinerea, E. lacerate (formerly Ceriporia lacerate), and I. lacteus and two cases of suspicious infection caused by $S$. commune and P. spadiceum. The Institutional Review Board of Seoul St. Mary's Hospital approved the study (approval no. KC19RESI0532) and waived the requirement to obtain informed consent from the patients because this is a retrospective study of clinical cases involving minimal risk to the patients.

The characteristics of the five cases and the fungal morphologies are presented in Table 1 and Fig. 1, respectively. They were all grown on Sabouraud dextrose agar after two weeks of incubation at $28^{\circ} \mathrm{C}$ under light. In all cases, the fungus was identified by sequencing the internal transcribed spacer (ITS)1/ITS2 and the 28S rRNA gene D1/D2 domains [4] using the following primer pairs: pITS1-F (5'-TCCGTAGGTGAACCTGCGG-3') and pITS1-R (5'-GCTGCGTTCTTCATCGATGC-3'); pITS2-F (5'-GCATCGATGAAGGCAGC-3') and pITS2-R (5'-TCCTCCGCTTATTATGC-3'); and D1/D2 regions-F (5'-GCATATCAATAAGCGGAAAAG-3') and D1/D2 regions-R (5'-GGTCCGTGTTTCAAG ACGG-3). Then, the sequences were analyzed using Basic Local Alignment Search Tool (www.ncbi.nlm.nih.gov/BLAST).

Antifungal susceptibility testing of the five isolates was retrospectively performed in triplicate according to the CLSI-M38
Received: January 21, 2019

Revision received: June 18, 2019

Accepted: August 7, 2019

Corresponding author: Yeon-Joon Park, M.D., Ph.D.

Department of Laboratory Medicine, College of Medicine, Seoul St. Mary's Hospital, Catholic University of Korea, 222 Banpo-daero, Seocho-gu, Seoul 06591, Korea

Tel: +82-2-2258-1640, Fax: +82-2-2258-1719, E-mail: yjpk@catholic.ac.kr

\section{(c) (i) (3)}

\section{(C) Korean Society for Laboratory Medicine}

This is an Open Access article distributed under the terms of the Creative Commons Attribution Non-Commercial License (http://creativecommons.org/licenses/by-nc/4.0) which permits unrestricted non-commercial use, distribution, and reproduction in any medium, provided the original work is properly cited. 


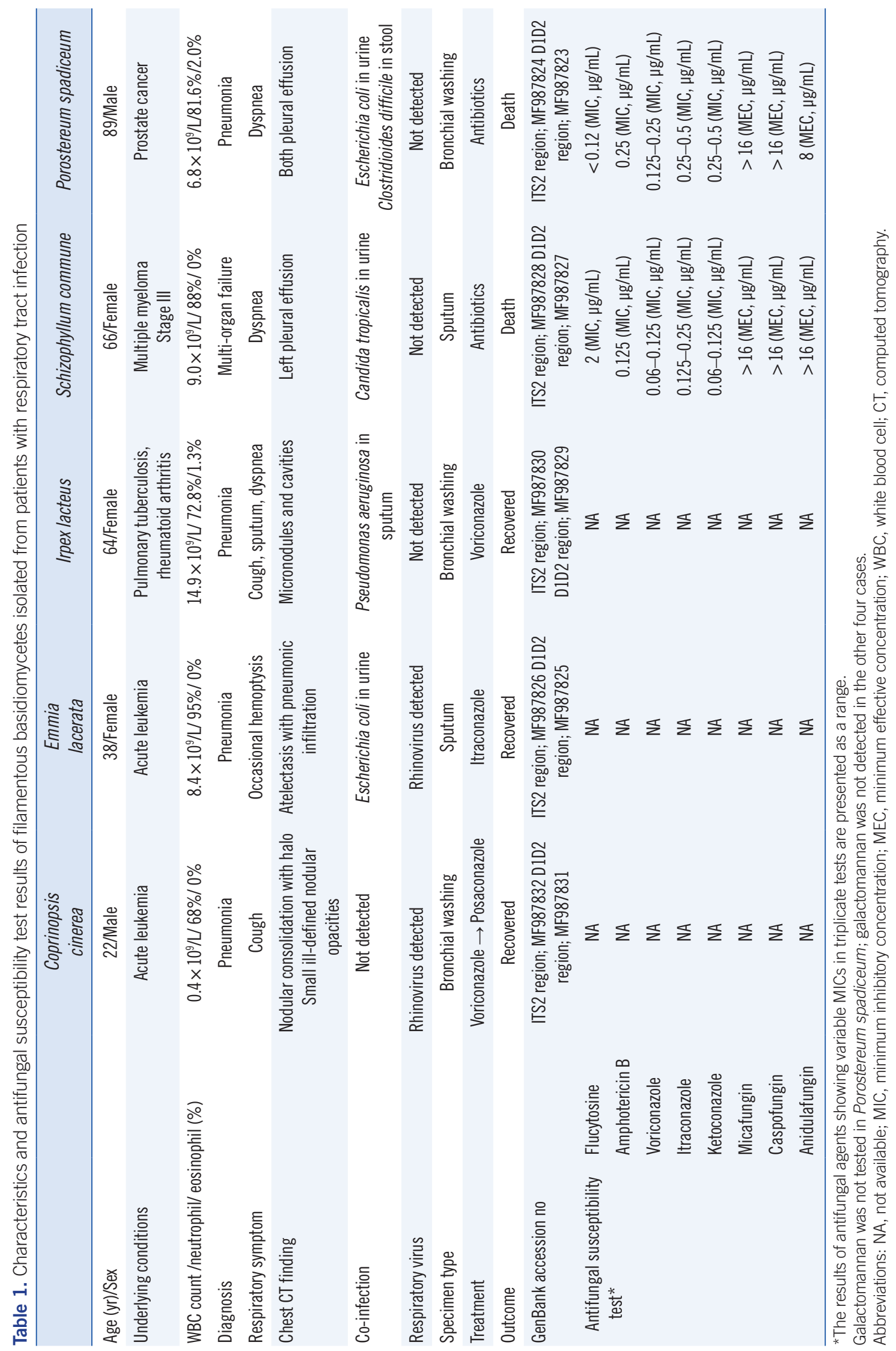



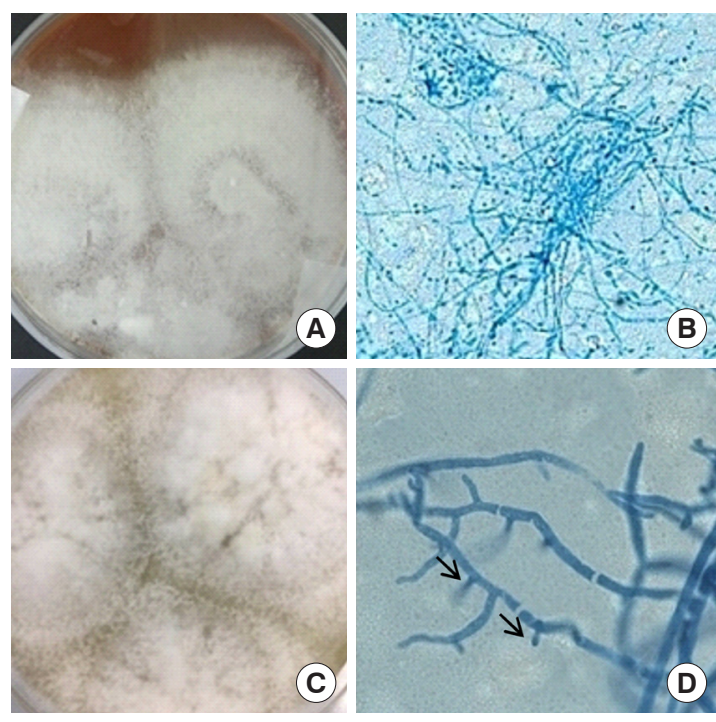

(c)
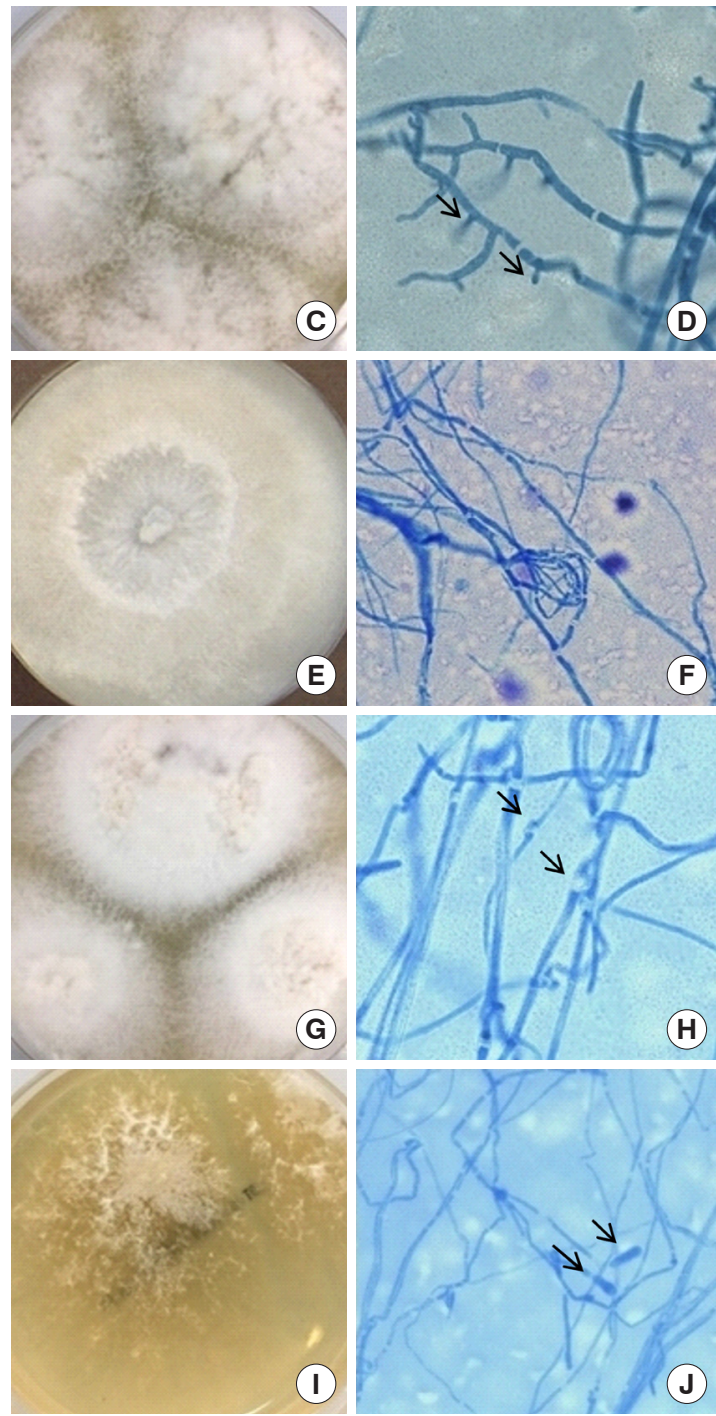

Fig. 1. White mold growth in five cases of basidiomycetes inoculated on Sabouraud dextrose agar (SDA) following incubation at $28^{\circ} \mathrm{C}$ under light for two weeks; lactophenol cotton blue mount of the mold slide culture on SDA at a magnification of $\times 400$. (A) White colony and $(B)$ hyaline hyphae with clusters of rectangular arthroconidia of Coprinopsis cinerea; (C) white colony and (D) spicules (arrows) of Emmia lacerata; (E) white colony of Irpex lacteus; (F) I. lacteus slide showing only hyaline hyphae; $(G)$ white colony and $(H)$ clamp connections (arrows) of Schizophyllum commune; (I) white colony of Porostereum spadiceum; (J) P. spadiceum showing enlarged hyphae (arrows). guidelines with a few modifications [5]. The isolates were cultured on potato dextrose agar for five days at $28^{\circ} \mathrm{C}$ and then shifted to $37^{\circ} \mathrm{C}$ incubation for five days for sporulation. The final inoculum of the homogenized fungal hyphae was adjusted to a density of $2.5-5.0 \times 10^{4}$ hyphal fragments $/ \mathrm{mL}$ by adjusting the optical density at $530 \mathrm{~nm}$ to $0.13-0.18$ using a spectrophotometer (VERSAmax microplate reader, Molecular Devices LLC, CA, USA). The microtiter plate was incubated at $35^{\circ} \mathrm{C}$ for 72 hours. Results were obtained only for $S$. commune and $P$. spadiceum. They showed low minimum inhibitory concentrations (MICs) for flucytosine, amphotericin B (AMB), voriconazole (VRC), itraconazole, and ketoconazole and high minimum effective concentrations for micafungin, caspofungin, and anidulafungin. For the remaining three isolates, we could not determine the MICs.

C. cinerea is normally found in compost and sewage; however, it rarely causes pulmonary infections, endophthalmitis, endocarditis, and chronic sinusitis [2]. A previous study reported an isolate susceptible to voriconazole and posaconazole but resistant to AMB, caspofungin, and micafungin [6]. Our patient also recovered from fungal pneumonia after treatment with voriconazole and posaconazole.

E. lacerate, an agent of white rot on wood, has recently been reported as a human pathogen with low MICs for azoles but high MICs for echinocandins [7, 8]. To our knowledge, this is the first clinical report of $E$. lacerate in Korea, and the patient recovered from fungal pneumonia after itraconazole treatment.

Another wood-decaying fungus, I. lacteus, has been rarely reported. A patient from Austria presenting with a pulmonary abscess was cured following treatment with voriconazole and AMB [9]. Our patient also showed improved chest computed tomography (CT) findings after treatment with voriconazole.

S. commune mainly causes sinusitis, allergic bronchopulmonary disease, fungal ball, and asthma. One Korean patient, who had a sino-orbital infection, was successfully cured using voriconazole and AMB [10], which is in line with our susceptibility test results.

P. spadiceum has also been isolated from respiratory specimens; however, its pathogenicity is yet to be established [2]. A study on antifungal susceptibility test using two isolates of $P$. spadiceum showed low MICs for AMB, voriconazole, and itraconazole [3], similar to our results.

As BM have been reported as human pathogens only recently and most of these reports mainly detail isolate characteristics, there are few clues to aid in the diagnosis of infection caused by these emerging pathogens. Of our five patients, three showed supportive chest CT findings, such as consolidation, nodular 
opacity, air-fluid level, and cavitation, while the other two patients showed only pleural effusions that were insufficient to support fungal pneumonia. However, we hypothesized that these two cases were fungal infections because the patients were immunocompromised, and the respiratory tract specimens were negative for acid-fast bacilli, aerobic pathogens, and respiratory viruses. In addition, S. commune is the most common BM that causes respiratory tract infections in immunocompromised patients. P. spadiceum was isolated from a bronchial wash specimen. Of note, the three patients treated with appropriate antifungal agents survived, while the other two patients who received only antibiotics died.

To our knowledge, this is the first report on respiratory infections caused by BMs in Korea. Although there was insufficient clinical evidence for $S$. commune and $P$. spadiceum infections, it is still important to identify these rare pathogens using molecular techniques to accumulate data. These efforts will help us better understand these infections and develop future diagnostic and therapeutic guidelines.

\section{Author Contributions}

All authors contributed equally to this study.

\section{Conflicts of Interest}

No potential conflicts of interest relevant to this article were reported.

\section{Research Funding}

This work was supported by a grant of the Korea Health Technology R\&D Project through the Korea Health Industry Development Institute, funded by the Ministry of Health \& Welfare, Republic of Korea (HI16C0443), and the Korea Medical Fungal Pathogen Resource Bank (KMFRB) under National Culture Collection for Pathogens (NCCP) of the Korea Center for Disease Control and Prevention (SPRB-2018-02).

\section{ORCID}

Jong-Mi Lee

https://orcid.org/0000-0003-2890-5285

Eunhee Han https://orcid.org/0000-0001-6519-1539

Jayoung Kim https://orcid.org/0000-0003-2977-1813

Ji-Hyun Park https://orcid.org/0000-0002-3002-6856

Gi-Ho Sung Jong Hee Shin https://orcid.org/0000-0002-1861-5543 https://orcid.org/0000-0001-9593-476X Yeon-Joon Park https://orcid.org/0000-0003-2182-5821

\section{REFERENCES}

1. De Pauw B, Walsh TJ, Donnelly JP, Stevens DA, Edwards JE, Calandra T, et al. Revised definitions of invasive fungal disease from the European Organization for Research and Treatment of Cancer/Invasive Fungal Infections Cooperative Group and the National Institute of Allergy and Infectious Diseases Mycoses Study Group (EORTC/MSG) Consensus Group. Clin Infect Dis 2008;46:1813-21.

2. Chowdhary A, Kathuria S, Agarwal K, Meis JF. Recognizing filamentous basidiomycetes as agents of human disease: a review. Med Mycol 2014; 52:782-97.

3. Singh PK, Kathuria S, Agarwal K, Gaur SN, Meis JF, Chowdhary A. Clinical significance and molecular characterization of nonsporulating molds isolated from the respiratory tracts of bronchopulmonary mycosis patients with special reference to basidiomycetes. J Clin Microbiol 2013; 51:3331-7.

4. CLSI. Interpretive criteria for identification of bacteria and fungi by DNA target sequencing. CLSI MM18-A. Wayne, PA: Clinical and Laboratory Standards Institute. 2007.

5. CLSI. Reference method for broth dilution antimicrobial susceptibility testing of filamentous fungi. Approved standard 3rd ed. CLSI M38. Wayne, PA: Clinical and Laboratory Standards Institute. 2017.

6. Abuali MM, Posada R, Del Toro G, Roman E, Ramani R, Chaturvedi S, et al. Rhizomucor variabilis var. regularior and Hormographiella aspergillata infections in a leukemic bone marrow transplant recipient with refractory neutropenia. J Clin Microbiol 2009;47:4176-9.

7. Chowdhary A, Agarwal K, Kathuria S, Singh PK, Roy P, Gaur SN, et al. Clinical significance of filamentous basidiomycetes illustrated by isolates of the novel opportunist Ceriporia lacerata from the human respiratory tract. J Clin Microbiol 2013;51:585-90.

8. Brandt ME. Filamentous basidiomycetes in the clinical laboratory. Curr Fungal Infect Rep 2013;7:219-23.

9. Buzina W, Lass-Flörl C, Kropshofer G, Freund MC, Marth E. The polypore mushroom Irpex lacteus, a new causative agent of fungal infections. J Clin Microbiol 2005;43:2009-11.

10. Sa HS, Ko KS, Woo KI, Peck KR, Kim YD. A case of sino-orbital infection caused by the Schizophyllum commune. Diagn Microbiol Infect Dis 2012;73:376-7. 